\title{
Calidad de vida relacionada con la salud del niño y del adolescente con obesidad
}

\author{
Jaime Gabriel Hurtado-Valenzuela, ${ }^{1}$ Gerardo Álvarez-Hernández²
}

Artículo original

\section{SUMMARY}

Objective

To estimate the health-related quality of life (HRQoL) in a group of obese children and adolescents from the city of Hermosillo, Sonora, Mexico.

\section{Methods}

A cross sectional study was performed using the PedsQL® questionnaire to compare the HRQoL of 200 children and adolescents with obesity as well as their parents, with two control groups: one from the community $(n=400)$ and another group of users $(n=200)$ from the Hospital Infantil del Estado de Sonora. The differences in HRQoL were assessed using a Kruskal-Wallis test and their relationship with sociodemographic variables and physical status was examined with logistic regression.

\section{Results}

HRQoL was lower in obese patients. Social and emotional QoL were lower in obese subjects. Perception of parents on HRQoL was lower than that of their children. $37.5 \%$ of obese subjects perceived their HRQoL as "very bad", which was lower than control groups. The risk of poor HRQoL was 2.2 times higher in the obese group.

\section{Conclusions}

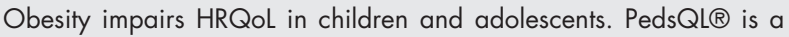
useful tool for the systematic assessment of HRQoL in children with obesity.

Key words: Quality of life related to health, obesity, children, adolescents.

\section{RESUMEN}

\section{Objetivo}

Estimar la calidad de vida relacionada con la salud (CVRS) en un grupo de niños y adolescentes con obesidad de la ciudad de Hermosillo, Sonora, México.

\section{Métodos}

Se realizó un estudio transversal utilizando el cuestionario PedsQL® para comparar la CVRS de 200 niños y adolescentes con obesidad al igual que a sus padres, con dos grupos de control, uno de la comunidad $(n=400)$ y otro grupo de usuarios $(n=200)$ del Hospital Infantil del Estado de Sonora. Las diferencias en la CVRS fueron evaluadas mediante una prueba de Kruskal-Wallis, y su relación con variables sociodemográficas y de estatus corporal se examinó con regresión logística.

\section{Resultados}

La CVRS fue menor en los sujetos obesos y las funciones más deterioradas fueron la emocional y la social; la percepción de los padres acerca de la CVRS fue inferior a la de sus hijos. El $37.5 \%$ de los sujetos obesos percibe su CVRS como "muy mala", una cifra inferior a los otros grupos. El riesgo de mala CVRS fue 2.2 veces mayor en el grupo de obesos.

\section{Conclusiones}

En nuestra población, la obesidad se asoció negativamente con la CVRS de niños y adolescentes. El PedsQL $®$ es un instrumento útil para la evaluación sistemática de la CVRS en niños con obesidad.

Palabras clave: Calidad de vida relacionada a la salud, obesidad, niños, adolescentes.

\section{INTRODUCCIÓN}

La obesidad es un problema mundial de salud pública. En los niños y adolescentes tiene un comportamiento epidémico pues para el año 2010 más de 43 millones de niños menores de cinco años tenían sobrepeso u obesidad, $35 \mathrm{mi}-$ llones de los cuales vivían en países en desarrollo. ${ }^{1}$ En los adolescentes, las cifras son menos claras, pero en algunos países industrializados la prevalencia oscila entre 9 y $18 \% .^{2}$
En México, en el año 2006, 9.4\% de los niños y 8.7\% de las niñas de cinco a 11 años eran obesos. Entre 1999 y 2006 se observó un alarmante incremento de 77\% (5.3 a 9.4\%) en los varones y de $47 \%$ ( 5.9 a $8.7 \%$ ) en las niñas. ${ }^{3}$

Múltiples factores han sido vinculados a su comportamiento, por lo que se ha recomendado diseñar soluciones preventivas e integrales. ${ }^{4}$ Aunque gran parte del interés científico en la obesidad pediátrica se ha centrado en sus efectos médicos, se reconoce una asociación inversa con as-

Hospital Infantil del Estado de Sonora. Licenciatura en Nutrición Humana, Universidad Estatal de Sonora.

2 Departamento de Medicina y Ciencias de la Salud, Universidad de Sonora.

Correspondencia: Dr. Jaime G. Hurtado-Valenzuela. Reforma Norte 355, colonia Ley, Hermosillo, Son, México. Tel.: (66) 2289 - 0600 ext. 80734. E-mail: jaimeghurtadov@gmail.com

Recibido primera versión: 23 de febrero de 2012. Segunda versión: 12 de julio de 2013. Tercera versión: 27 de noviembre de 2013. Aceptado: 2 de diciembre de 2013 
Hurtado-Valenzuela y Álvarez-Hernández

pectos funcionales y psicosociales de niños y adolescentes, lo que deteriora su calidad de vida.,

Para examinar tal relación, se ha acuñado el concepto de calidad de vida relacionada a la salud (CVRS), que se refiere a la forma en que una persona percibe su salud física y mental. La CVRS se ha usado para medir los efectos de las enfermedades crónicas y la forma en que éstas interfieren en la vida cotidiana del individuo. ${ }^{7}$ Diversas investigaciones han documentado la relación entre la CVRS de niños y adolescentes, con padecimientos como obesidad, cáncer, asma, diabetes y fibrosis quística. ${ }^{7-10}$

Uno de los instrumentos más utilizados para medir la CVRS en niños y adolescentes con diferentes patologías, agudas o crónicas, es el Cuestionario Pediátrico sobre Calidad de Vida (PedsQL $®$, por sus siglas en inglés). ${ }^{11}$ El PedsQL 4.0® tiene diversas adaptaciones idiomáticas, incluida una para México. El cuestionario permite calificar de forma estandarizada la percepción de la CVRS en distintas enfermedades, lo que puede proveer información relevante a tomadores de decisiones clínicas y de salud pública. ${ }^{11,12}$

Aunque se reconoce que una de las consecuencias tempranas de la obesidad en la edad pediátrica son las alteraciones psicológicas y sociales, poco se ha investigado al respecto en México. El objetivo del presente estudio fue documentar la relación entre la obesidad y la CVRS en un grupo de escolares de la ciudad de Hermosillo, lo que puede contribuir a identificar efectos tempranos de la obesidad sobre la CVRS, y ayudar al diseño de estrategias preventivas y de atención de este problema.

\section{MÉTODOS}

Previa aprobación por la Secretaría de Educación y Cultura del Estado de Sonora y el Comité de Ética del Hospital Infantil del Estado de Sonora (HIES), se realizó este estudio con un diseño transversal. Se comparó una muestra probabilística de niños y adolescentes con obesidad, con dos grupos de la población y sus respectivos padres. El grupo de casos fue conformado por sujetos de ocho a 15 años de edad, y sus padres, que asistieron durante el año 2008 a la Clínica de Obesidad y Nutrición del HIES. Esta muestra se comparó con dos grupos comparados por el método de pares por edad, el primero conformado por individuos seleccionados aleatoriamente de escuelas primarias y secundarias públicas de la ciudad, inscrito durante el ciclo 2008-2009. El segundo grupo incluyó niños y adolescentes que acudieron a consulta externa del HIES por una patología distinta a la de interés del estudio; en ambos grupos se incluyó a su padre o madre. En todos los sujetos se obtuvo la firma del consentimiento informado por parte del familiar responsable y de los sujetos seleccionados.

Se excluyeron pacientes con obesidad secundaria a alguna causa médica y sujetos con obesidad que hubieran recibido tratamiento para su problema. Se eliminó el $4 \%$ de los pacientes con información incompleta o inadecuada en el cuestionario, y/o que se negaron a la realización de la somatometría. Una vez elegido, en cada binomio padremadre/niño-adolescente se recolectó información acerca de la edad, sexo, escolaridad, peso y talla, estado marital del informante y ocupación del principal sostén económico de la familia. Se realizó antropometría consistente en la toma del peso corporal y la talla tanto a los niños y adolescentes como a su padre o madre.

\section{Tipo de muestreo y tamaño de la muestra}

Se estima que en Sonora $16 \%$ de los niños y adolescentes presentan obesidad, ${ }^{13}$ y considerando que el HIES atiende anualmente en la consulta ambulatoria a 4000 pacientes, se definió un tamaño de muestra de 200 sujetos con obesidad, con una confianza de $95 \%$. Para seleccionar al grupo escolar se utilizó un muestreo polietápico usando el marco muestral de las escuelas primarias y secundarias públicas del sitio de estudio. De ellas, se eligió aleatoriamente a 10 escuelas primarias y cinco secundarias, estratificadas por estatus socioeconómico. Al interior de las escuelas, se escogió aleatoriamente a los grupos de escolares que participaron. La etapa final incluyó la selección aleatoria de 400 participantes de entre los distintos grupos. El grupo de controles hospitalarios $(n=200)$ fue elegido aleatoriamente de pacientes que acudieron por diversos motivos a consulta externa del HIES, excepto por problemas nutricionales. En los tres grupos de estudio se incluyó al padre, madre o tutor del sujeto seleccionado.

\section{Instrumentos de medición}

Para la medición de la CVRS se utilizó el cuestionario PedsQL 4.0®, que comprende dos formatos, uno para la respuesta del niño o adolescente y otro para sus padres, con lo que se obtiene la percepción del binomio. Las preguntas de ambos formatos son las mismas pero difieren en su construcción gramatical y están validados para las edades investigadas. ${ }^{11,12}$ La instrucción básica es responder a la pregunta: ¿qué tanto problema tuve en el último mes? Una escala de cinco puntos califica las respuestas: $0=$ nunca; $1=$ casi nunca; $2=$ a veces; 3 =frecuentemente; $4=$ casi siempre. Las respuestas son codificadas en forma inversa y transformadas linealmente a una escala de $0-100(0=100,1=75,2=50,3=25,4=0)$, por lo que una calificación alta significa mejor CVRS. ${ }^{12,13}$ Con la distribución percentilar del puntaje se crearon cinco estratos de CVRS: muy mala (por abajo del percentil 20); mala (entre el 20 y 40); regular (entre el 40 y 60); buena (entre el 60 y 80), y muy buena (arriba del percentil 80). El cuestionario se aplicó de forma independiente, en un cubículo separado, al sujeto de estudio y su tutor. La aplicación al grupo de escolares se realizó en el salón de clases. 


\section{Somatometría}

Los participantes fueron pesados en una báscula electrónica de piso, marca Seca modelo 813. La estatura se tomó con un estadiómetro portátil, marca Seca modelo 214. Las mediciones fueron realizadas por tres asistentes de nutrición; las de los niños obesos y los controles de la consulta externa se hicieron en el consultorio, el grupo de escolares se midió y pesó en su escuela. La somatometría se realizó con la técnica habitual, con ropa de uso diario y sin zapatos. Los mismos procedimientos e instrumentos se usaron para las mediciones de los padres participantes. El Índice de Masa Corporal (IMC) fue empleado para categorizar el estatus corporal de los participantes; se clasificó como obesidad un IMC arriba de $30 \mathrm{~kg} / \mathrm{m}^{2}{ }^{14}$ Se utilizaron los percentiles del IMC para niños y niñas de la Organización Mundial de la Salud y que recomienda las Guías de Práctica Clínica Mexicanas ${ }^{15}$ para formar los estratos de obesidad (arriba de percentil 95), sobrepeso (entre los percentiles 85 y 95) y peso normal (entre los percentiles 5 y 85).

\section{Análisis estadístico}

Mediante estadística descriptiva se caracterizó a los sujetos del estudio. Las diferencias entre los distintos estratos de CVRS fueron examinadas mediante la prueba de KruskalWallis y, para no sobrestimar el valor de p debido a las múltiples comparaciones, se realizaron ajustes con el método de Bonferroni. La variable respuesta (CVRS) fue dicotomizada (Mala CVRS=percentil $\leq 60$; Buena $C V R S=$ percentil $\geq 61$ ) para examinar su relación con el peso corporal ( $1=$ obeso), sexo (1=masculino), escolaridad ( $1=$ primaria) y edad $(1=8 \mathrm{a}$ 10 años), mediante análisis logístico bivariado y multivaria- do. El paquete estadístico utilizado fue el Number Cruncher Statistical System (NCSS®) 2007 versión 7.1.17.

\section{RESULTADOS}

De la población estudiada, los varones $(n=418)$ fueron la mayor proporción (52.0\%), aunque sin diferencia $(\mathrm{p}=0.127)$ respecto a las mujeres. Los niños de ocho a 10 años constituyeron la mayor proporción $(44.8 \%)$, apreciándose diferencias $(\mathrm{p}<0.01)$ cuando se le comparó con los grupos de 11 a $13(35 \%)$ y de 14 a 15 años (20\%). Con respecto a la escolaridad, $534(66.8 \%)$ sujetos asistían a escuelas primarias y 266 $(33.2 \%)$ a secundarias $(\mathrm{p}<0.01)$.

La prevalencia de obesidad del total de los grupos fue de $40.8 \%$, mientras que la de sobrepeso fue de $10.8 \%$. Veinte ( $2.5 \%)$ de los sujetos tenían bajo IMC para su edad y sexo, en tanto otros $368(46.0 \%)$ tenían talla y peso normales.

En general, los hombres tuvieron mayor prevalencia de exceso de peso (33.2\%) que las mujeres $(18.4 \%)(p<0.001)$. El peso promedio de los sujetos del grupo con obesidad fue de $72.2 \mathrm{~kg}$, mientras los controles del hospital (43.5 kg) y escolares $(47.9 \mathrm{~kg}$ ) fue menor $(\mathrm{p}<0.01)$. De forma similar, se observó mayor talla e IMC en el grupo con obesidad $(p<0.01)$. Por lo que respecta a la edad, los niños de ocho a 10 años tuvieron la prevalencia (22.0\%) más alta con sobrepeso u obesidad. Entre los informantes, no se apreciaron diferencias en la edad (media=37.2 años), pero el IMC (media=32.3) y peso promedio $(82.3 \mathrm{~kg})$ fueron mayores en el grupo de padres de niños obesos $(\mathrm{p}<0.0001)$ (cuadro 1).

Las madres fueron el principal informante $(83 \%$ de las ocasiones), en tanto que los padres lo fueron en el 7.5\%. Los padres del grupo escolar tenían mayor nivel de escolaridad

Cuadro 1. Distribución de la edad y somatometría de los sujetos de estudio y sus padres. Hermosillo, Sonora, México. 2010

\begin{tabular}{|c|c|c|c|c|}
\hline \multirow[b]{2}{*}{ Variable } & \multicolumn{4}{|c|}{ Media (Desviación estándar) } \\
\hline & Obesos & Escolares & HIES a & $\mathrm{P} 1 /$ \\
\hline Niños & $(n=200)$ & $(n=400)$ & $(n=200)$ & \\
\hline - Edad en años & $11.60 \quad(2.30)$ & $10.80 \quad(2.20)$ & $11.40 \quad(2.30)$ & 0.001 \\
\hline - Peso en kg. & $72.20(20.20)$ & $43.50(15.70)$ & $47.90(19.20)$ & $<0.001$ \\
\hline - Talla en mt. & $1.52(0.12)$ & $1.46(0.14)$ & $1.50(0.15)$ & $<0.001$ \\
\hline - Índice de masa corporal* & $30.70 \quad(5.20)$ & $20.00 \quad(4.60)$ & $20.80 \quad(5.80)$ & $<0.001$ \\
\hline Informantes & $(n=200)$ & $(n=300)$ & $(n=200)$ & \\
\hline - Edad en años & $38.80 \quad(7.00)$ & $37.40 \quad(7.90)$ & $37.50 \quad(8.50)$ & 0.102 \\
\hline - Peso en kg. & $82.30(18.50)$ & $73.70(16.40)$ & $74.90(15.60)$ & 0.001 \\
\hline - Talla en mt. & $1.59(0.07)$ & $1.62(0.08)$ & $1.61 \quad(0.07)$ & 0.002 \\
\hline - Índice de masa corporal & $32.30 \quad(6.30)$ & $29.10 \quad(5.50)$ & $29.00 \quad(5.90)$ & $<0.001$ \\
\hline
\end{tabular}


Hurtado-Valenzuela y Álvarez-Hernández

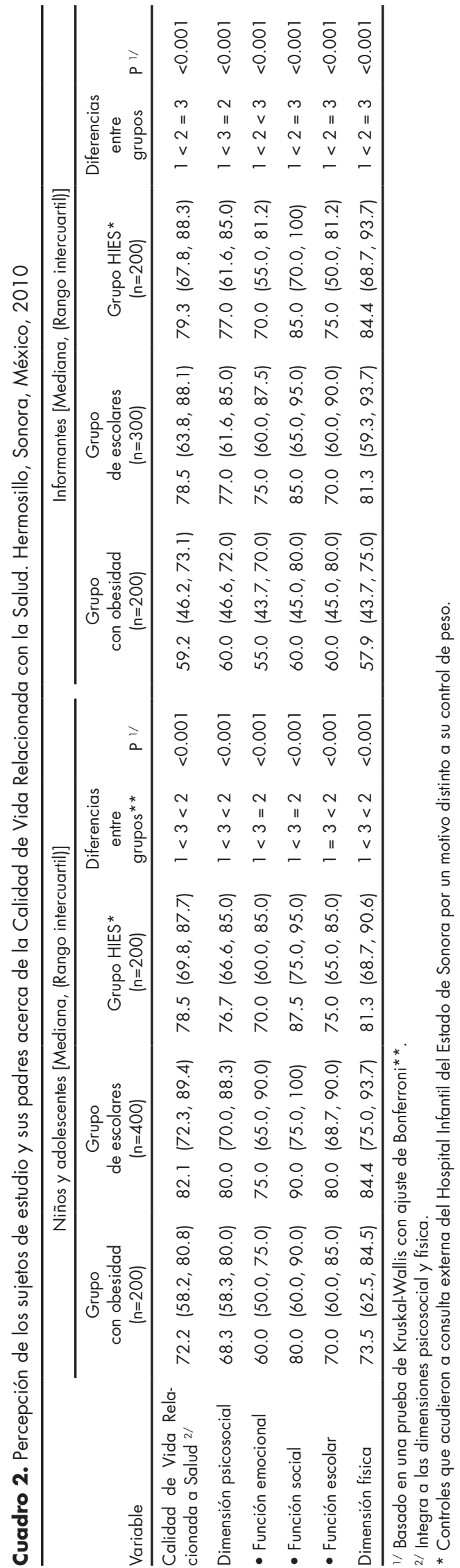

que los otros dos grupos $(\mathrm{p}=0.0097)$. Por lo que toca a la ocupación, los padres del grupo con obesidad laboran principalmente $(40.5 \%)$ en trabajos calificados, mientras que en los grupos, tanto de escolares como del Hospital, los padres reportaron desempeñarse con mayor frecuencia en diversos oficios $(\mathrm{p}<0.001)$.

Por lo que respecta a la CVRS, no observamos diferencias de acuerdo con el sexo de los sujetos de estudio $(p=0.2579)$. En cuanto a los sujetos con obesidad, éstos alcanzaron una puntuación mediana de 72.2 sobre una máxima de 100, y fue inferior a la de los otros dos grupos $(\mathrm{p}<0.001)$, tanto en la dimensión psicosocial (mediana $=68.3$ ) como en la física (mediana=73.5). De modo semejante, los padres de los sujetos con obesidad percibieron un mayor deterioro en la CVRS de sus hijos respecto de los otros dos grupos $(p<0.001)$; esta percepción fue similar tanto para aspectos psicosociales como físicos. Las calificaciones otorgadas por los informantes a las funciones emocional y física fueron incluso inferiores a las de sus hijos (cuadro 2).

Cuando la CVRS fue estratificada de acuerdo con su distribución percentilar, $37.5 \%$ de los sujetos con obesidad fueron clasificados con una "muy mala" CVRS, constituyendo la proporción más alta de la muestra y diferente de los grupos controles: 12 y 19\%, respectivamente $(p=0.0021)$. En el estrato de "mala" y "regular" CVRS no hubo diferencias entre los tres grupos. Asimismo, el estatus de "muy buena" CVRS sólo fue alcanzado por $6.5 \%$ de los sujetos con obesidad, contra el 26.2 y $19.0 \%$ de los grupos de control $(\mathrm{p}=0.0003)$ (cuadro 3). En los informantes se observó un comportamiento semejante. Así, $39.5 \%$ de los padres del grupo de obesos ubicó a sus hijos en el estrato de "muy mala" CVRS, contra únicamente el $14.3 \%$ y el $9.5 \%$ de los dos grupos restantes $(\mathrm{p}<0.001)$. Sólo $4.5 \%$ de los padres del grupo de obesos percibió como "muy buena" la CVRS de sus hijos, contra 24.3 y $26.5 \%$ de los otros dos grupos ( $<<0.001$ ) (cuadro 3 ).

$\mathrm{Al}$ examinar la relación bivariada de la CVRS con las variables estudiadas, se observó que los sujetos con "mala" CVRS estuvieron 2.1 veces más expuestos a obesidad (RM=2.13, IC95\% $[1.57,2.91])$. No se encontró que la CVRS estuviera asociada linealmente con la edad, el género o la escolaridad de los niños o adolescentes (cuadro 4), ni con la ocupación o escolaridad de sus padres (datos no mostrados). Cuando se ajustó en un modelo multivariado, el efecto de la obesidad sobre la CVRS se mantuvo (RMa $=2.24$, IC95\% $[1.64,3.06])$, no así con el sobrepeso (RMa $=0.85$, IC95\% [0.51, 1.41]) (cuadro 5).

\section{DISCUSIÓN}

Nuestros hallazgos muestran que la CVRS de niños y adolescentes con obesidad se encuentra más deteriorada que la de sus contrapartes sin obesidad. Esto es consistente con reportes previos ${ }^{6,8,16} \mathrm{y}$ sugiere la pertinencia de evaluar esferas 
Cuadro 3. Percepción de la Calidad de Vida Relacionada con la Salud (CVRS), de sujetos de estudio y sus padres, de acuerdo a categorías basadas en el cuestionario PedsQL®. Hermosillo, Sonora, México. 2010

\begin{tabular}{|c|c|c|c|c|c|c|c|c|}
\hline \multirow[b]{2}{*}{ Estrato de CVRS } & \multicolumn{4}{|c|}{ Niños y adolescentes [No. $(\%)]$} & \multicolumn{4}{|c|}{ Informantes [No. (\%)] } \\
\hline & $\begin{array}{c}\text { Grupo } \\
\text { con obesidad } \\
(n=200)\end{array}$ & $\begin{array}{c}\text { Grupo } \\
\text { de escolares } \\
(n=400)\end{array}$ & $\begin{array}{l}\text { Grupo HIES * } \\
\qquad(n=200)\end{array}$ & $\mathrm{P}^{1 /}$ & $\begin{array}{c}\text { Grupo } \\
\text { con obesidad } \\
(n=200)\end{array}$ & $\begin{array}{c}\text { Grupo } \\
\text { de escolares } \\
(n=300)\end{array}$ & $\begin{array}{l}\text { Grupo HIES * } \\
\qquad(n=200)\end{array}$ & $\mathrm{P}^{1 /}$ \\
\hline Muy mala & 75 (37.5) & $48(12.0)$ & $38(19.0)$ & 0.0021 & $79(39.5)$ & $43(14.3)$ & $19 \quad(9.5)$ & 0.0000 \\
\hline Mala & $47(23.5)$ & 75 (18.8) & $40(20.0)$ & NS & $53(26.5)$ & 54 (18.0) & $34(17.0)$ & 0.0308 \\
\hline Regular & $39(19.5)$ & $81(20.2)$ & 38 (19.0) & NS & $37(18.5)$ & $54(18.0)$ & $43(21.5)$ & NS \\
\hline Buena & $26(13.0)$ & $91(22.8)$ & $46(23.0)$ & 0.0134 & $22(11.0)$ & $73(24.3)$ & $51(25.5)$ & 0.0003 \\
\hline Muy buena & $(6.5)$ & $105(26.2)$ & 38 (19.0) & 0.0003 & $9 \quad(4.5)$ & $76(24.3)$ & $53(26.5)$ & 0.0000 \\
\hline
\end{tabular}

1/ Basado en una prueba multinominal de Chi-cuadrada para diferencia de proporciones.

NS = No significativo. CVRS = Calidad de Vida Relacionada a la Salud.

* Controles que acudieron a consulta externa del Hospital Infantil del Estado de Sonora por un motivo distinto a su control de peso.

adicionales a la clínica cuando se estudia la obesidad. Si bien hay un deterioro global de la CVRS de los niños y adolescentes con obesidad, la esfera psicosocial está más afectada e indicaría la necesidad de adicionar al manejo médico una atención multidisciplinaria del sujeto obeso, algo que ya ha sido señalado en reportes previos que incluyeron niños sin atención médica y gravemente obesos. ${ }^{8,17}$ También se ha documentado que el deterioro en la esfera psicosocial afecta de modo particular la autoestima del menor y en cómo se relaciona con sus pares, independientemente de su estrato socioeconómico y condición racial o étnica. ${ }^{18}$

Por otra parte, en nuestro estudio $60 \%$ de los padres con obesidad otorgaron una menor puntuación a la CVRS que sus hijos, particularmente en las esferas emocional y física, algo ya documentado antes. ${ }^{8,19}$ Una explicación que se ha dado a este hecho es que el propio exceso de peso de los padres los orilla a minimizar el efecto que el sobrepeso puede ocasionar en la salud de sus hijos. ${ }^{16,20,21}$ Otros reportes $^{22}$ han sugerido que incluso las psicopatologías de los pa- dres pueden confundir o modificar la percepción que tienen sobre la CVRS de sus hijos y consecuentemente afectar el cumplimiento de indicaciones médicas, nutricionales y psicológicas para resolver el problema.

Justo por las discrepancias que se observan en las valoraciones que hacen padres e hijos de la CVRS es que se han propuesto estrategias para mejorar la confiabilidad de los cuestionarios que la miden, sea por procedimientos de autoevaluación o por "proxis", usualmente los padres. Los resultados son aún inciertos, pues mientras algunos estudios reportan baja concordancia entre los hijos y sus padres, ${ }^{23}$ otros señalan un acuerdo que oscila desde moderado hasta sustancial. ${ }^{8,24}$ Estudios futuros en México que incorporen la diversidad geográfica y cultural del país pueden examinar las potenciales diferencias en la percepción de la CVRS entre padres e hijos, controlando el efecto de variables socioculturales.

Aunque no observamos que la edad modificara la relación entre obesidad y CVRS, es posible que el riesgo de mala

Cuadro 4. Factores de riesgo para Mala Calidad de Vida Relacionada a la Salud en niños y adolescentes de Hermosillo, Sonora, México. 2010. Resultados de la regresión logística bivariada

\begin{tabular}{|c|c|c|c|c|}
\hline Variable & Coeficiente $\beta$ & Error estándar & $\begin{array}{c}\text { Razón } \\
\text { de momios 1/ }\end{array}$ & $95 \%$ IC RM \\
\hline \multicolumn{5}{|c|}{ Peso corporal (1 = Normal) } \\
\hline - Obesidad & 0.758 & 0.157 & 2.13 & $(1.57,2.91)$ \\
\hline - Sobrepeso & -0.163 & 0.258 & 0.85 & $(0.51,1.41)$ \\
\hline \multicolumn{5}{|c|}{ Sexo (1 = Masculino) } \\
\hline - Femenino & 0.182 & 0.144 & 1.20 & $(0.90,1.59)$ \\
\hline \multicolumn{5}{|c|}{ Escolaridad (1 = Primaria) } \\
\hline - Secundaria & 0.222 & 0.154 & 1.25 & $(0.92,1.69)$ \\
\hline \multicolumn{5}{|c|}{ Edad ( $1=8$ a 10 años) } \\
\hline - 11 a 13 años & 0.429 & 0.164 & 1.54 & $(1.11,2.12)$ \\
\hline - 14 a 15 años & 0.236 & 0.193 & 1.27 & $(0.87,1.85)$ \\
\hline
\end{tabular}


Hurtado-Valenzuela y Álvarez-Hernández

Cuadro 5. Factores de riesgo para Mala Calidad de Vida Relacionada con la Salud en niños y adolescentes de Hermosillo, Sonora, México. 2010. Resultados de la regresión logística multivariada

\begin{tabular}{|c|c|c|c|c|}
\hline Variable & Coeficiente $\beta$ & Error estándar & $\begin{array}{c}\text { Razón de momios } \\
\text { ajustada }{ }^{1 /}\end{array}$ & $95 \%$ IC RM \\
\hline \multicolumn{5}{|l|}{$\begin{array}{l}\text { Peso corporal } \\
\text { (1.0 = Peso normal) }\end{array}$} \\
\hline - Obesidad & 0.805 & 0.159 & 2.24 & $(1.64,3.06)$ \\
\hline - Sobrepeso & -0.168 & 0.260 & 0.85 & $(0.51,1.41)$ \\
\hline - Bajo peso & 1.103 & 0.459 & 3.01 & $(1.23,7.41)$ \\
\hline Obesidad $x$ edad 1 & 0.108 & 0.306 & 1.11 & $(0.55,2.25)$ \\
\hline Obesidad $x$ edad 2 & -0.738 & 0.630 & 0.48 & $(0.14,1.64)$ \\
\hline Obesidad $x$ edad 3 & 0.623 & 0.434 & 1.86 & $(0.25,14.1)$ \\
\hline
\end{tabular}

CVRS ocurra desde edades tempranas, algo que se ha mencionado en reportes previos..$^{24,25}$ Nos llama la atención que no hubo diferencias en la CVRS de niños de ocho a 10 años y jóvenes de 14 y 15 años, dado que se ha propuesto que en algunos grupos de adolescentes hay cierta inestabilidad psicosocial que pudiera deteriorar la CVRS. ${ }^{26}$

No encontramos asociación con otras variables socioeconómicas que merecen estudios más específicos, como el estado civil, escolaridad materna, estatus socioeconómico y ocupación de los padres, algo similar a reportes previos. ${ }^{18}$ Esto no significa que no exista tal asociación, pues ya ha sido documentado ${ }^{27}$ que tal relación es no sólo posible, sino que sesgos de selección (p.ej., sólo incluimos población de un nivel socioeconómico relativamente bajo, de una misma región geográfica, de un hospital público, entre otros factores) pudieran ocultar o mitigar potenciales relaciones. Nuevos estudios regionales son convenientes, porque la relación de CVRS y obesidad infantil pudiera estar mediada por la clase social paterna. Esto ha sido observado en algunos grupos como los afroamericanos y méxicoamericanos, ${ }^{26,28}$ evidenciando que, aun en regiones afluentes, los estratos pobres de la población son los más afectados. ${ }^{27} \mathrm{El}$ efecto de clase social puede estar mediado tanto por factores ambientales y conductuales como por variables psicosociales.

Por otro lado, la mayoría de los estudios que han utilizado PedsQL $\AA$ categorizan binariamente a la CVRS - p.ej., como teniendo afección o una menor CVRS-, esto último cuando la puntuación alcanzada se aleja una desviación estándar. ${ }^{12,29}$ Nosotros proponemos que al estratificar las calificaciones mediante cuartiles se puede conferir un grado cualitativo a la percepción de la CVRS. Esta estratificación parece apropiada dado que la calificación de PedsQL® deriva en una escala numérica discreta. ${ }^{11}$ En consecuencia, sugerimos que los resultados del cuestionario se analicen mediante técnicas estadísticas no paramétricas, algo que, hemos observado, se utiliza poco en los reportes publicados. ${ }^{24,29}$
Así, se apreció que uno de cada tres sujetos con obesidad tiene una muy mala CVRS, mientras que un porcentaje muy bajo (6.5\%) pudo clasificarse como con muy buena CVRS. Este comportamiento fue distinto al de los otros dos grupos, de tal forma que la CVRS se deteriora al incrementarse el peso corporal especialmente en los sujetos que presentan ya el problema de obesidad. En relación con lo anterior, quedaría pendiente analizar la CVRS en los diferentes grados de obesidad, lo cual sería tema para un futuro reporte. No obstante lo anterior, recomendamos cautela en la interpretación de los hallazgos debido a las características de la muestra y al diseño del estudio, por lo que parece conveniente investigar la CVRS a nivel comunitario, lo que contribuiría a la construcción de indicadores positivos de salud y no solamente a estimar el efecto de ciertas enfermedades pediátricas tanto agudas como crónicas. ${ }^{18,30,31}$

Finalmente, a pesar del posible sesgo debido a la selección de un grupo hospitalario de pacientes obesos, los resultados son consistentes con otros reportes y corroboran que la obesidad deteriora la CVRS de los niños y adolescentes, particularmente la esfera emocional, y se aprecia que la obesidad duplica el riesgo de tener una mala CVRS en escolares de la ciudad de Hermosillo, Sonora. En este sentido, el cuestionario PedsQL® en su versión para México es un instrumento válido y de fácil aplicación para examinar la relación de obesidad y CVRS. Los resultados nos indican que la CVRS puede evaluarse sistemáticamente en niños y adolescentes con obesidad, y que nuevos estudios contribuirían a ampliar el conocimiento de investigadores, personal de salud y directivos acerca de los problemas prioritarios de salud pública regionales.

\section{REFERENCIAS}

1. World Health Organization. Population-based prevention strategies for childhood obesity. Report of the WHO Forum and Technical Meeting. Ginebra: 2009. 
2. Ogden CL, Yanovsky SZ, Carroll MD, Flegal KM. The epidemiology of obesity. Gastroenterol 2007;132:2087-2102.

3. Olaiz-Fernández G, Rivera-Dommarco J, Shamah-Levy T, Rojas R et al. Encuesta Nacional de Salud y Nutrición 2006. Cuernavaca, México: Instituto Nacional de Salud Pública; 2006.

4. Gordon-Larsen P, Nelson MC, Page P, Popkin BM. Inequality in the built environment underlies key health disparities in physical activity and obesity. Pediatr 2006;117(2):417-424.

5. Fallon EM, Tanofsky-Kraff M, Norman AC, McDuffe JR et al. Health-related quality of life in overweight and nonoverweight black and white adolescents. J Pediatr 2005;147:443-450.

6. Tsiros MD, Olds T, Buckley JD, Grimshaw P et al. Health-related quality of life in obese children and adolescents. Int J Obes 2009;33:387-400.

7. Sawyer MG, Reynolds KE, Couper JJ, French DJ et al. Health-related quality of life of children and adolescents with chronic illness -a two year prospective study. Qual Life Res 2004;13:1309-1319.

8. Pinhas-Hamiel O, Singer S, Pilpel N, Fradkin A et al. Health-related quality of life among children and adolescents: associations with obesity. Int J Obes 2005;30(2):267-72.

9. Matziou V, Perdikaris P, Feloni D, Moshovi C et al. Cancer in childhood: Children's and parent's aspects for quality of life. European J Oncology Nursing 2008;12:209-216.

10. Van Gent R, Van der Ent CK, Rovers MM, Kimpen JL et al. Excessive body weight is associated with additional loss of quality of life in children with asthma. J Allergy Clin Immunol 2007;119(3):591-596.

11. Varni JW, Seid M, Kurtin PS. PedsQL ${ }^{8} 4.0$ Reliability and validity of the pediatric Quality of Life Inventory Version 4.0 Generic Core Scales in Healthy and Patients Populations. Med Care 2001;39:800-812.

12. Varni JW, Burwinkle TM, Seid M, Skarr D. The PedsQL® 4.0 as a pediatric population health measure: Feasibility, reliability, and validity. Ambul Pediatr 2003;3:329-341.

13. Hurtado-Valenzuela JG, Sotelo-Cruz N, Avilés-Rodríguez M, Peñuelas-Beltrán CI. Aumento en la prevalencia de obesidad en niños y adolescentes de la consulta ambulatoria. Bol Clin Hosp Infant Edo Sonora 2005;22(2): 81-86.

14. Cole TJ, Bellizi MC, Flegal KM, Dietz WH. Establishing a standard definition for child overweight and obesity worldwide: international survey. BMJ 2000;320:1240-1243.

15. Prevención y Diagnóstico de Sobrepeso y Obesidad en Niños y Adolescentes en el Primer Nivel de Atención. Guía de Práctica Clínica. Consejo de Salubridad General 2012. México. Consultado en línea en octubre 2013. http://www.cenetec.salud.gob.mx/

16. Kim D, Kawachi I. Obesity and health-related quality of life. Chapter 12. Part II. Epidemiologic studies of consequences of obesity. En: Hu FB (ed.). Obesity epidemiology. Cambridge: Oxford Press; 2008; pp 234-260.
17. Schwimmer JB, Burwinkle TM, Varni JW. Health-related quality of life of severely obese children and adolescents. JAMA 2003;289(14):1813-1819.

18. Wallander JL, Kerbawy S, Toomey S, Lowry R et al. Is obesity associated with reduced health-related quality of life in Latino, black and white children in the community? Int J Obes 2013 doi:10.1038/ ijo.2013.31.

19. Wake M, Salmon L, Waters E, Wright $M$ et al. Parent-reported health status of overweight and obese Australian primary school children: a cross-sectional population survey. Int J Obesity 2002;26:717-724.

20. Baughcum AE, Chamberlain LA, Deeks CM, Powers SW et al. Maternal perceptions of overweight preschool children. Pediatrics 2000;106(6):1380-1386.

21. Strauss RS. Childhood obesity. Pediatr Clin North Am 2002;49:175-201.

22. Steinsbekk S, Josefiak T, Ødegård R, Wichstrøm L. Impaired parent-reported quality of life in treatment-seeking children with obesity is mediated by high levels of psychopatology. Qual Life Res 2009;18:1159-1167.

23. Theunissen NCM, Vogels TGC, Koopman HM. The proxy problem: Child report versus parent report in health-related quality of life research. Qual Life Res 1998;7:387-397.

24. Hughes AR, Farewell K, Harris D, Reilly JJ. Quality of life in a clinical simple of obese children. Int J Obes 2007;31(1):39-44.

25. Sato H, Nakamura M, Sasaki N. Effects of bodyweight on health-related quality of life in school-aged children and adolescents. Pediatr Int 2008;50:552-556.

26. Tyler Ch, Johnston CA, Fullerton G, Foreyt JP. Reduced quality of life in very overweight Mexican-American adolescents. J Adolesc Health 2007;40:366-368.

27. Gearhart RF, Gruber DM, Vanata DF. Obesity in the lower socioeconomic status segments of the American society. Forum in public policy. Ashland University, Ohio USA. Serie en internet $2008 \mathrm{http} / / / \mathrm{www}$. forumonpublicpolicy.com/archivespring08/gearhart.pdf. [Consultado el 23 de octubre de 2011]

28. Zeller MH, Modi AC. Predictors of health quality of life in obese youth. Obesity 2006;14(1):122-130.

29. Varni JW, Limbers CA, Burwinkle TM. Impaired health-related quality of life in children and adolescents with chronic conditions: a comparative analysis of 10 disease clusters and 33 disease categories/severities utilizing the PedsQL 4.0 Generic Core Scales. Health Quality Life Outcomes 2007, doi:10.1186/1477/7525-5-43.

30. Simon AE, Chan KS, Forrest CB. Assessment of Children's HealthRelated Quality of Life in the United States with a Multidimensional Index. Pediatrics 2008;121(1):118-126.

31. Strauss RS, Knight J. Influence of the home environment on the development of obesity in children. Pediatrics 1999;103(6):e85.

Artículo sin conflicto de intereses 